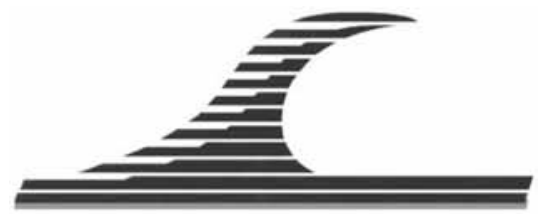

Revue Paralia, Volume 4 (2011) pp 7.1-7.12

Mots-clés : Lagune, Morphologie, Bathymétrie, Anthropisation, Dynamique sédimentaire, Etangs palavasiens, Etang de l'Or.

(C) Editions Paralia CFL

\title{
Evolution morphologique récente d'un complexe lagunaire méditerranéen : le système des étangs Palavasiens (France)
}

\author{
Jérôme CASTAINGS ${ }^{1,2}$, Laurent DEZILEAU ${ }^{1}$, \\ Annie FIANDRINO ${ }^{2}$, Romaric VERNEY ${ }^{3}$
}

1. Laboratoire Géosciences Montpellier, UMR 5243 - CC 60 - Université Montpellier 2, Place E. Bataillon, 34095 Montpellier cedex 5, France.

2. Ifremer, Laboratoire Environnement-Ressources Languedoc-Roussillon, Bd Jean Monnet, BP 171, 34203 Sète Cedex, France.

jerome.castaings@ifremer.fr

3. Ifremer, Laboratoire de Physique Hydrodynamique et Sédimentaire - DYNECO, BP 70, 29280, Plouzané, France.

\section{Résumé :}

Les lagunes du Golfe du Lion constituent un patrimoine naturel reconnu ainsi qu'un enjeu essentiel pour les activités économiques locales. Leur évolution morphologique est donc une question importante dans le contexte actuel de changements climatiques et environnementaux. L'analyse de données topo-bathymétriques du système des étangs Palavasiens a permis de mieux cerner son évolution depuis le XVIII ${ }^{\text {ème }}$ siècle. Les actions anthropiques et les apports du bassin versant apparaissent comme les facteurs principaux de l'évolution du système. Au cours des 40 dernières années, le système montre une tendance à se combler lentement avec une accumulation moyenne de 1,3 mm/an. Des différences de fonctionnement sur chacun des étangs sont mises en lumière en fonction des forçages naturels et anthropiques locaux.

Soumis le 13 mai 2011, accepté le 27 septembre 2011, en ligne le 31 octobre 2011. La seule version examinée est celle écrite en français. La ou les autres versions n'étant pas examinées par le comité de rédaction de la revue, sont donc publiées sous l'entière responsabilité du ou des auteurs.

A TRANSLATED VERSION IN ENGLISH IS AVAILABLE ONLINE

Pour citer cet article :

CASTAINGS J., DEZILEAU L., FIANDRINO A., VERNEY R. (2011). Evolution morphologique récente d'un complexe lagunaire méditerranéen : le système des étangs Palavasiens (France). Revue Paralia, Vol. 4, pp 7.1-7.12. 


\section{Introduction}

Les milieux lagunaires sont reconnus pour être des systèmes en équilibre instable sur le plan morphologique et peuvent tendre à terme soit vers un comblement soit vers une érosion ou une submersion marine (PHLEGER, 1969 ; NICHOLS, 1989 ; BIRD, 1994). Ces environnements sont soumis à des apports sédimentaires divers d'origine terrestre, marine, endogène (production biologique) et éolienne (CASTAINGS, 2008). La diversité de ces facteurs et la variabilité des forçages naturels et anthropiques s’exerçant sur ces milieux rendent leur évolution difficile à déterminer. Pourtant, le devenir morphologique des lagunes est une question essentielle pour la gestion de ces espaces naturels et pour l'avenir des activités humaines qui y sont liées (pêche, conchyliculture, navigation, tourisme...). Le devenir de différents systèmes lagunaires a été récemment étudié de par le monde : Laguna Madre (MORTON et al., 2000), bassin d'Arcachon (ALLARD et al., 2009), lagune de Venise (MOLINAROLI et al., 2009). A travers l'analyse de données topographiques et bathymétriques, nous essaierons de retracer les tendances évolutives d'un complexe lagunaire dont l'évolution reste encore mal connue, le système des étangs Palavasiens situé au sud de la France dans l'Hérault (figure 1a).

\section{Présentation du site d'étude}

Les lagunes du Golfe du Lion se sont formées au cours de la période Holocène sous l'influence de l'hydrodynamisme local et des variations du niveau marin (RAYNAL et al., 2010). Depuis, elles constituent un piège pour les sédiments qui s'y accumulent (MARTIN, 1978 ; CATALIOTTI-VALDINA, 1978 ; DUBOUL-RAVAZET \& MARTIN, 1981 ; BARUSSEAU et al., 1992). Le complexe lagunaire des étangs Palavasiens, situé au Nord du Golfe du Lion, s'étend sur une trentaine de kilomètres (figure 1a). A l'origine, le système constituait une seule lagune, reliée également à l'étang de Thau à l'Ouest comme l'attestent les cartographies anciennes (ANDREOSSY, 1669 ; CASSINI, 1778). Il a été fragmenté par les dynamiques naturelles et les aménagements successifs. La canalisation du Lez vers la mer (XVII ${ }^{\text {ème }}$ siècle) et le creusement du Canal de navigation du Rhône à Sète (fin du XVIII ${ }^{\text {ème }}$ et $\mathrm{XIX}^{\mathrm{ème}}$ siècle) ont particulièrement contribué à cette séparation. La première partie de ce canal est visible sur la carte de Cassini (figure 1b). De nos jours, le complexe lagunaire est formé de neufs étangs principaux. Les apports sédimentaires vers ce système lagunaire sont irréguliers et fortement dépendants des conditions climatiques méditerranéennes. Le débit des cours d'eau est faible voire inexistant durant la période estivale, les apports sédimentaires se concentrent essentiellement lors des crues rapides (BOURRIN et al., 2006) ; cependant peu de données quantitatives sont disponibles sur ces petits bassins versants. Les apports sédimentaires marins peuvent s'effectuer par submersion des points bas du cordon littoral en période de tempête entraînant l'ouverture de graus temporaires (DEZILEAU et al., 2010) ou bien à travers des graus artificialisés maintenus ouverts en permanence. Le système a subi une urbanisation 
Evolution morphologique récente d'un complexe lagunaire méditerranéen : le système des étangs Palavasiens (France) : 7.3

importante au cours du dernier siècle avec notamment le développement de l'agglomération de Montpellier et l'essor des stations touristiques implantées sur le cordon littoral dans les années 1970 (Frontignan-Plage, Palavas-les-Flots, Carnon, La Grande Motte).

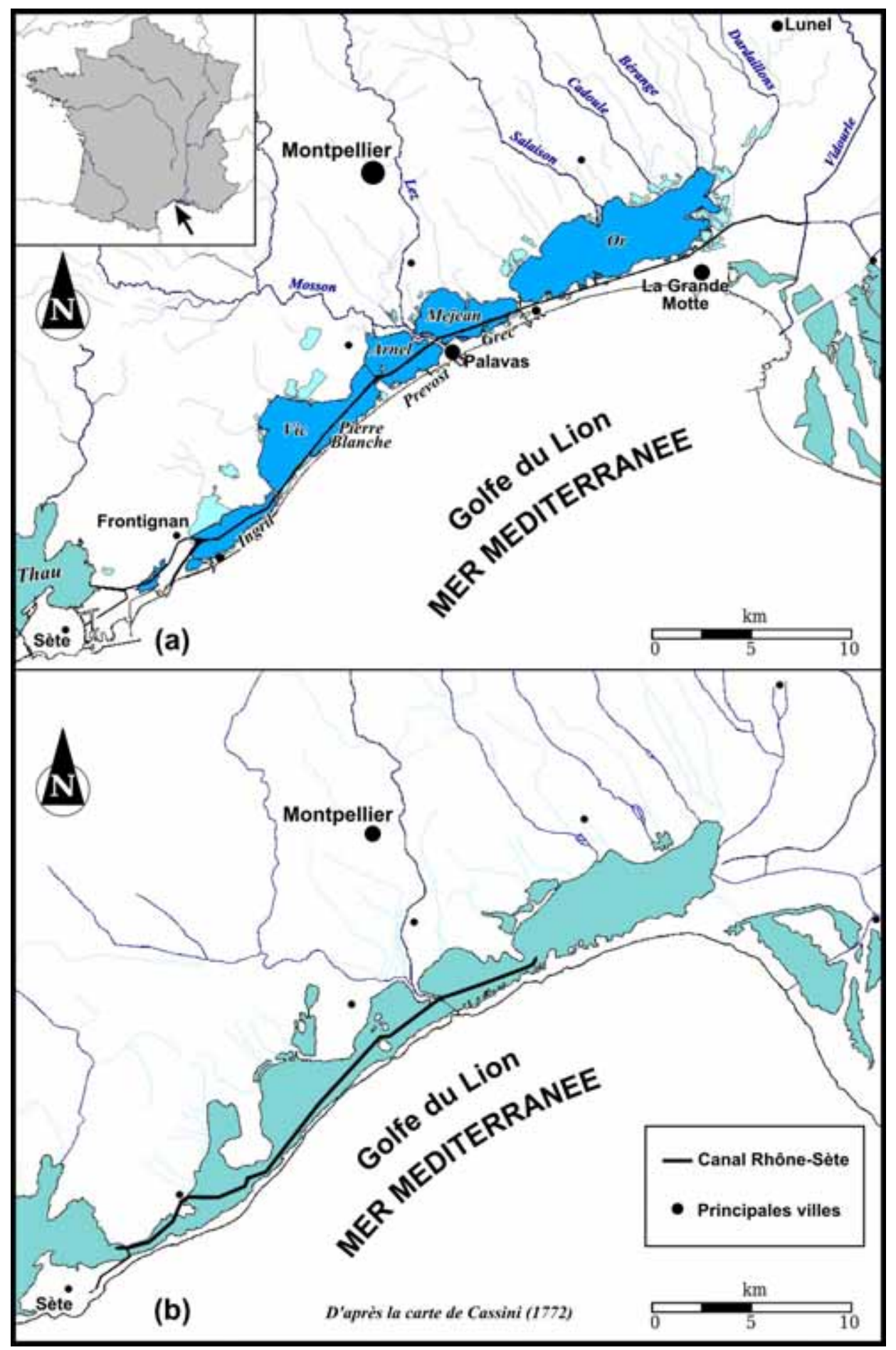

Figure 1. Localisation du site d'étude (a) et situation au XVIII ${ }^{\text {ème }}$ siècle (b). 


\section{Matériel et méthode}

\subsection{Données utilisées et corrections}

Deux types de données ont été utilisés pour cette étude, des données topographiques du pourtour des lagunes (période 1772-2005) et trois jeux de données bathymétriques (période 1965-2005) comme cela est résumé dans le tableau 1.

Le contour des lagunes en 1772 a été numérisé à partir d'une carte de Cassini calée localement sur 200 points fixes (principalement des églises et mas persistants à l'époque actuelle) à l'aide du logiciel QGIS. La précision moyenne généralement admise pour la carte de Cassini est de l'ordre de 300 mètres (EHESS/CNRS \& BNF, 2004); cette valeur s'accorde avec l'erreur moyenne du calage effectué qui est de 284 mètres. Le contour et la bathymétrie des lagunes pour les séries 1965 et 1985 ont été numérisés à partir de cartes papier d'échelle 1/10000 et d'une table à digitaliser GTCO CalComp. Pour l'année 2005, le trait de côte utilisé est issu de la BDTOPO de l'Institut Géographique National.

Les données bathymétriques de 1965 sont constituées de profils de points de sonde orientés globalement Nord-Sud et espacés de 100 à 500 mètres selon les étangs. La précision de la méthode d'acquisition bathymétrique de l'époque n’est pas connue de même que celle du trait de côte des étangs. La série de données des années 1980 inclus uniquement les étangs de Pierre-Blanche (1980), Grec (1986), Or (1989) et Prévost (1991), leur précision en altitude est de $\pm 10 \mathrm{~cm}$. Le jeu de données 2005 est constitué de profils orientés perpendiculairement à l'axe du canal du Rhône à Sète espacés de 100 à 200 mètres ainsi que de données plus resserrées au niveau des passes entre les étangs et le Canal du Rhône à Sète. Les sondes ont été acquises à l'aide d'un GPS différentiel couplé à un sondeur mono-faisceau avec une précision de $\pm 4 \mathrm{~cm}$ en altitude (SMNLR, 2006).

Tableau 1. Données topo-bathymétriques utilisées.

\begin{tabular}{|c|c|c|c|}
\hline Date & Source & Contour & Bathymétrie \\
\hline 1772 & $\begin{array}{l}\text { Carte de Cassini feuilles } n \circ 57 \text { et } 92 \\
\text { acquisition entre } 1770 \text { et } 1774\end{array}$ & Ensemble de la zone & Non disponible \\
\hline 1965 & $\begin{array}{l}\text { CNABRL Mission d'aménagement du Littoral } \\
\text { acquisition entre } 1964 \text { et } 1966\end{array}$ & Ensemble de la zone & Ensemble de la zone \\
\hline 1985 & SMNLR - acquisition entre 1980 et 1991 & Données partielles & Données partielles \\
\hline 2005 & SMNLR - acquisition entre 2004 et 2006 & Ensemble de la zone & Ensemble de la zone \\
\hline
\end{tabular}

L'ensemble des données ont été converties au format de coordonnées Lambert 93. Le système IGN69 est utilisé comme référence altimétrique, les données de 1965 basées sur le système Lallemand ont été converties. Par ailleurs, afin d'éviter les effets dus à la 
variation du niveau marin sur le calage du niveau de base, les données ont été corrigées. L'élévation du niveau marin au cours du dernier siècle est estimée entre 1 et $4 \mathrm{~mm} / \mathrm{an}$ (GIEC, 2007 ; CABANES et al., 2001). Le niveau en Méditerranée semble augmenter moins rapidement que les océans (CALAFAT \& GOMIS, 2009). Il présente cependant des disparités spatiales avec une augmentation plus importante dans le Golfe du Lion que sur la côte provençale (CAZENAVE et al., 2002). Au regard de ces différentes études, nous avons donc choisi d'appliquer une correction des données bathymétriques anciennes basée sur une élévation moyenne du niveau marin de $2 \mathrm{~mm} / \mathrm{an}$. La correction a été calculée séparément pour chaque lagune en fonction des dates précises des jeux de données bathymétriques.

\subsection{Evolution de la surface lagunaire}

Les contours de chacune des lagunes ont été séparés et ont fait l'objet d'un calcul de surface pour les dates 1772, 1965 et 2005. Les surfaces ont ensuite été comparées entre elles pour évaluer leur évolution. Seuls les neufs plans d'eau principaux ont été considérés dans cette étude, les marais salants et les étangs mineurs (surface inférieure à 100 ha) représentant au total environ $10 \%$ de la surface de la zone d'étude ne sont pas pris en compte. En effet, ils sont alors déconnectés du système global et les observations de terrain montrent qu'ils sont le plus souvent asséchés en été. L'incertitude sur la surface lagunaire a été calculée à partir de la précision des données originelles pour 2005 et à partir des erreurs de calage pour les cartes numérisées de 1772 et 1965. De plus, pour 1772 sur les secteurs présentant peu de points fixes pour le calage, la surface des marais bordant l'étang a été prise en compte pour majorer l'incertitude.

\subsection{Evolution bathymétrique des lagunes}

Les données bathymétriques ont été traitées à l'aide du logiciel ArcGIS et Spatial Analyst. Les points de sonde ont été interpolés séparément pour chaque lagune sur une grille avec une résolution de $50 \times 50 \mathrm{~m}$. Ce calcul est basé sur un algorithme de pondération par l'inverse de la distance (IDW) dont les paramètres ont été ajustés en fonction de la densité des données pour chaque date disponible. Les fichiers matriciels résultants (rasters) ont été comparés entre eux. Pour cela, l'outil Raster Calculator a été utilisé en effectuant les opérations algébriques [2005-1965], [2005-1985] et [19851965]. Cette opération a permis de différencier les zones de dépôt, de stabilité et d'érosion. Afin de prendre en compte l'incertitude importante des données anciennes nous avons choisi une approche conservative (VAN DER WAL \& PYE, 2003) en représentant comme stables les variations bathymétriques inférieures à $\pm 25 \mathrm{~cm}$. 


\section{Résultats et discussions}

\subsection{Evolution générale du système}

Les résultats de l'évolution surfacique des étangs (tableau 2 et figure 2) montrent une tendance générale à la réduction des milieux lagunaires. Cependant, il est possible de distinguer deux groupes d'étangs. Les étangs du Grec, Ingril, Pierre Blanche et Prévost présentent une nette diminution de leur surface en eau. Les étangs de l'Or, Vic, Arnel et Méjean présentent un caractère plus stable. La disparition est plus rapide sur la période récente avec une perte moyenne de 8,2 ha/an depuis 1965 contre 4,8 ha/an sur la période antérieure. L'évolution bathymétrique (figures 3 et 4) montre une tendance générale au comblement du système avec une part importante de zones stables liées à l'approche conservative adoptée.

Tableau 2. Surface lagunaire actuelle et évolution relative depuis 1772.

\begin{tabular}{llll}
\hline Lagune & Surface actuelle (ha) & Evolution 1772-1965 & Evolution 1965-2005 \\
\hline Ingril & 534 & $-38 \pm 12 \%$ & $-11 \pm 3 \%$ \\
Vic & 1337 & $-5 \pm 10 \%$ & $-1 \pm 0,5 \%$ \\
Pierre-Blanche & 270 & $-17 \pm 11 \%$ & $-11 \pm 4 \%$ \\
Arnel & 445 & $-10 \pm 10 \%$ & $+1 \pm 0,5 \%$ \\
Prévost & 245 & $-8 \pm 10 \%$ & $-9 \pm 4 \%$ \\
Méjean & 727 & $-3 \pm 10 \%$ & $0 \pm 0,5 \%$ \\
Grec & 117 & $-39 \pm 12 \%$ & $-33 \pm 9 \%$ \\
Or & 3039 & $-6 \pm 10 \%$ & $-4 \pm 2 \%$ \\
\hline
\end{tabular}

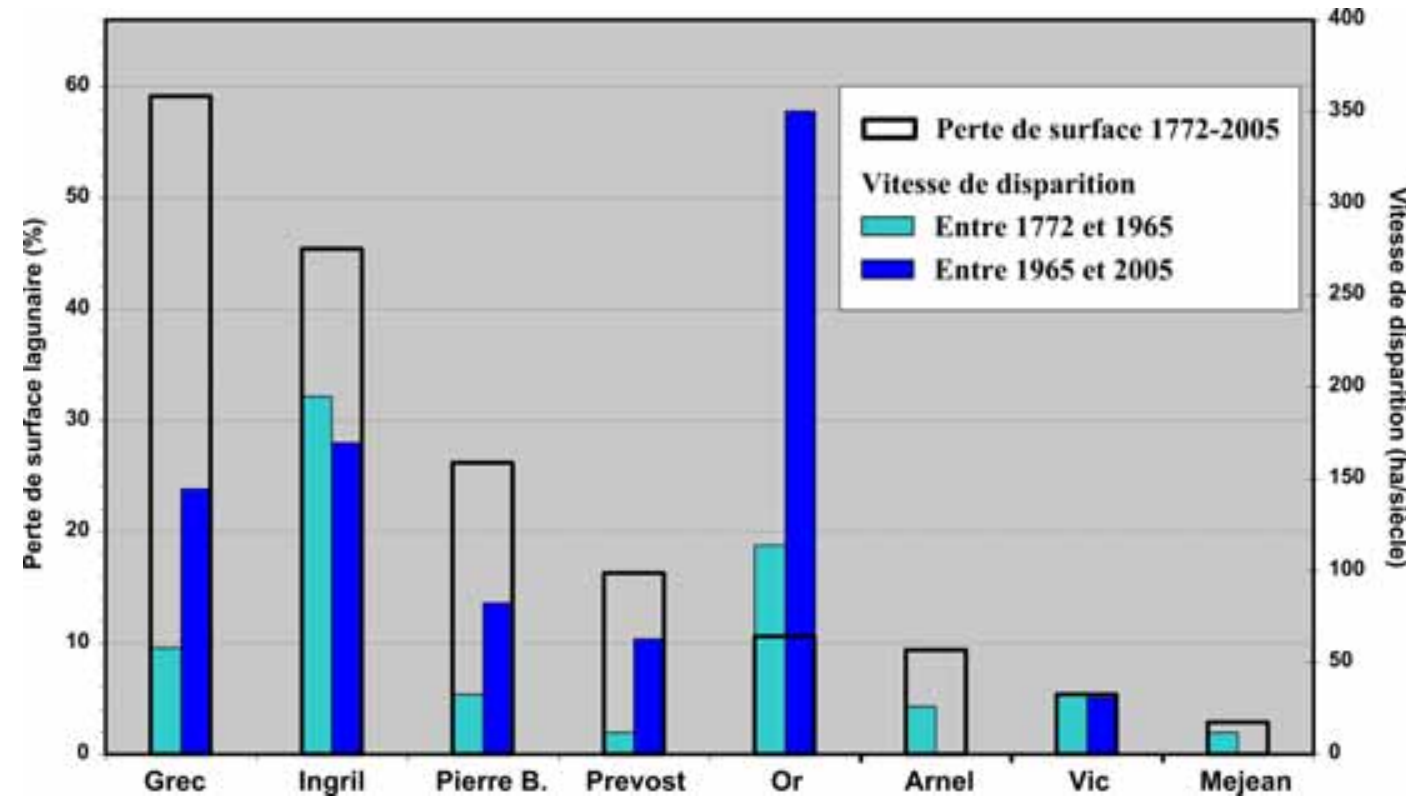

Figure 2. Perte de surface des étangs Palavasiens. 


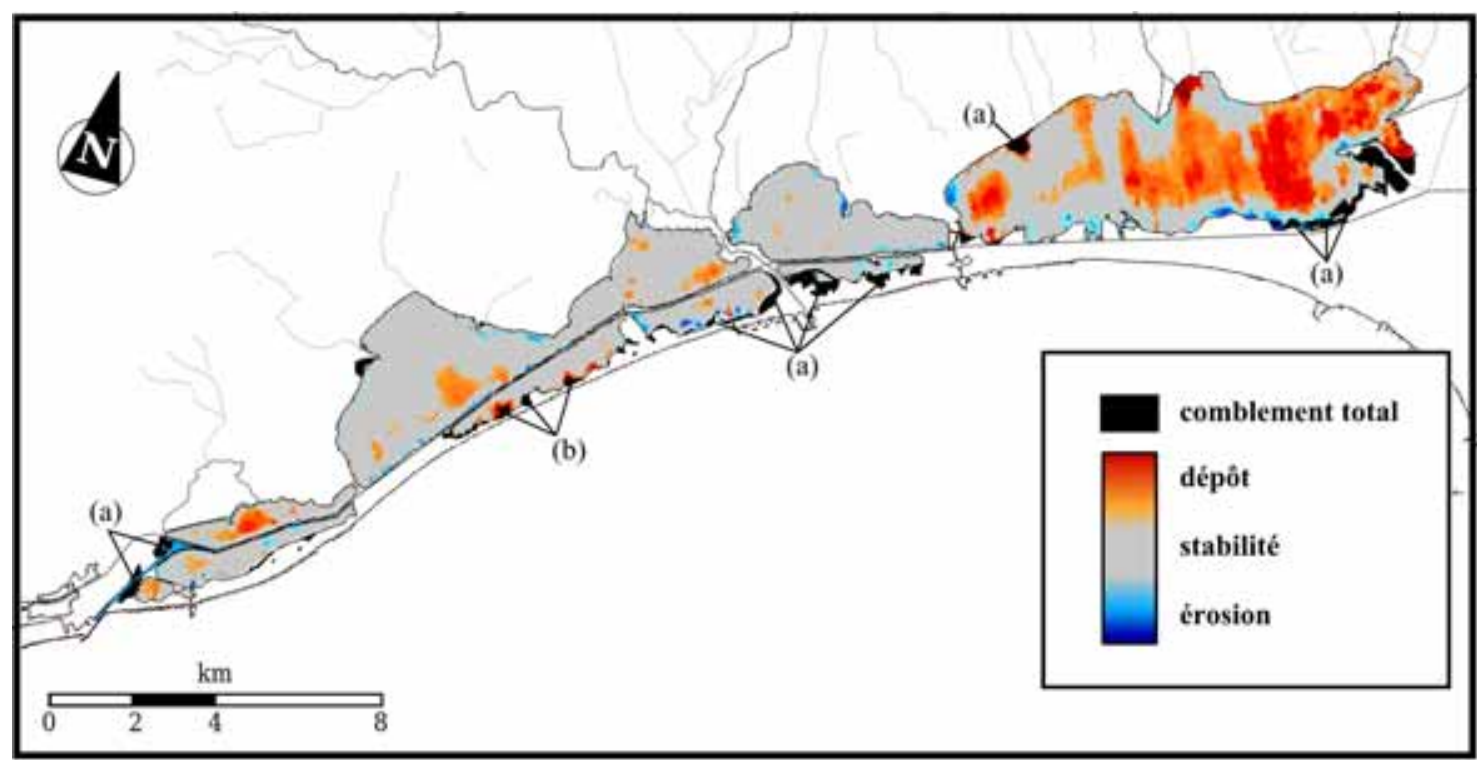

Figure 3. Evolution des étangs Palavasiens entre 1965 et 2005

Les principales zones comblées sont d'origine anthropique (a) et naturelle (b).

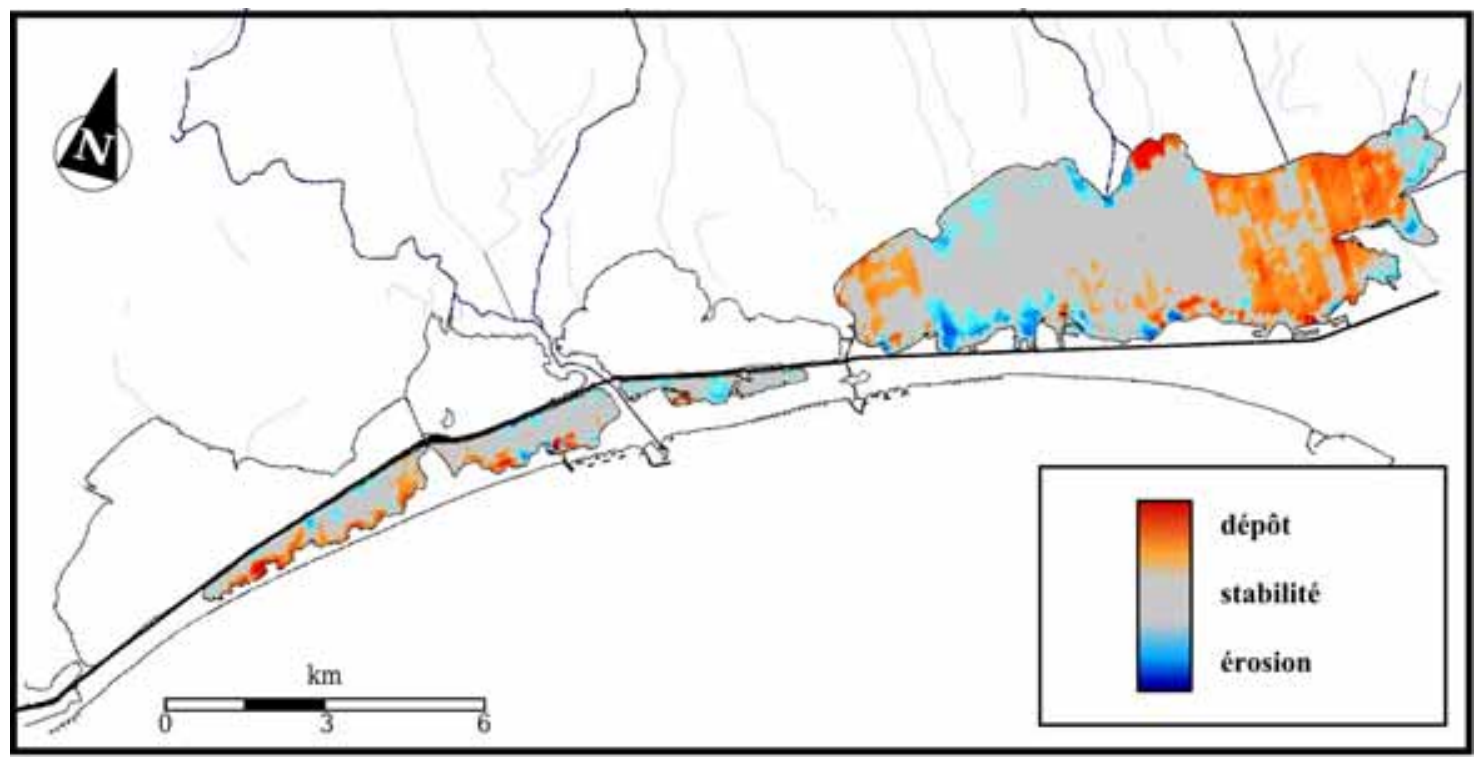

Figure 4. Evolution bathymétrique des étangs Palavasiens entre 1985 et 2005.

\subsection{Interprétation des évolutions surfaciques}

Une étude de l'occupation actuelle des sols permet d'identifier des causes anthropiques comme étant à l'origine de la plupart des zones entièrement comblées au cours de la période récente (figure 3). Sur l'étang d'Ingril les modifications sont anciennes avec la séparation des Salins de Frontignan. Le comblement se poursuit avec le développement de la zone industrialo-portuaire de Sète-Frontignan au cours du $\mathrm{XX}^{\text {ème }}$ siècle, la construction d'une zone de tourisme balnéaire (années 1970) et l'aménagement d'un nouveau chenal de dérivation du Canal du Rhône à Sète (années 1980). 
Les étangs du Prévost et du Grec sont directement affectés par le développement urbain et touristique de la zone littorale tout au long du $\mathrm{XX}^{\text {ème }}$ siècle et surtout depuis les années 1970.

L'étang de Pierre-Blanche présente, lui aussi, une accélération du comblement sur la période récente. Cependant, il s'agit vraisemblablement ici de cônes sédimentaires d'origine naturelle liés à la dynamique marine durant les évènements climatiques extrêmes. Ce processus est probablement renforcé par divers facteurs : la fragilisation du cordon dunaire liée à une forte fréquentation estivale, l'absence d'aménagements de protection des plages contrairement aux zones adjacentes et l'élévation du niveau marin. Compte tenu de sa surface importante, l'étang de l'Or présente une évolution relative limitée, cependant son évolution est une des plus rapides (figure 2). Il est comblé initialement par la progradation sédimentaire des cours d'eau. Au cours du dernier demi-siècle le comblement s'accélère en raison de l'action anthropique, notamment l'extension de l'aéroport de Montpellier-Fréjorgues au Nord ainsi que par l'aménagement de connexions hydrauliques avec le canal du Rhône à Sète au Sud.

La surface des étangs de Vic, Arnel et Méjean évolue peu sur la période considérée. La canalisation du Lez vers la mer (XVII ${ }^{\text {ème }}$ siècle) a certainement ralenti le comblement de ces étangs en déviant les apports sédimentaires.

\subsection{Interprétation des évolutions bathymétriques}

Au sein des lagunes, la principale zone de dépôt est située dans la partie Est de l'étang de l'Or, secteur où se concentrent de nombreux apports de cours d'eau (Cadoule, Bérange, Dardaillons et divers canaux dont des connexions avec le Vidourle). Les autres secteurs de dépôts importants se localisent dans les étangs de l'Arnel, Vic, PierreBlanche et Ingril. Pour l'étang de l'Arnel, les dépôts sont vraisemblablement liés à des apports de crues de la Mosson et du Lez. Dans l'étang de Vic, les dépôts se concentrent dans la zone la plus profonde de la lagune, cette accumulation préférentielle peut s'expliquer par un plus faible remaniement des sédiments par les vagues. Pour l'étang de Pierre-Blanche, les dépôts se localisent principalement dans la continuité de zones entièrement comblées par les cônes de tempêtes. Ces dépôts constituent un facteur de comblement reconnu également sur des époques antérieures pour cette lagune (SABATIER, 2009). Pour l'étang d'Ingril, le nombre important de communications avec le canal du Rhône à Sète (six passes contre une à trois pour les autres étangs) pourrait constituer une source pour les dépôts constatés. L'étang du Prévost présente des zones de dépôt réduites qui sont localisées à proximité des apports externes : passe avec le Canal du Rhône à Sète, Canalette de Palavas et le grau. Le comblement sur cet étang est sous-estimé car des dragages ont été effectués sur la période considérée pour désensabler le grau et ses abords. Enfin, les étangs du Méjean et du Grec ne présentent quasiment pas de zones en dépôt. 
Les secteurs en érosion sont localisés sur les bordures des étangs. Le faible niveau d'eau y permet une remise en suspension plus importante du sédiment lors de coups de vent. Les dragages peuvent également contribuer à cette évolution. Sur la période étudiée, des dragages ont été réalisés au niveau des passes entre les étangs et le Canal du Rhône à Sète, au niveau des graus ainsi que dans l'étang du Prévost.

Un point remarquable est celui de la pointe du Salaison (figure 5). Suite à la construction d'un nouveau chenal d'évacuation des crues, l'ancienne avancée sédimentaire est aujourd'hui en érosion et une zone de dépôt se forme à la sortie du nouveau chenal.

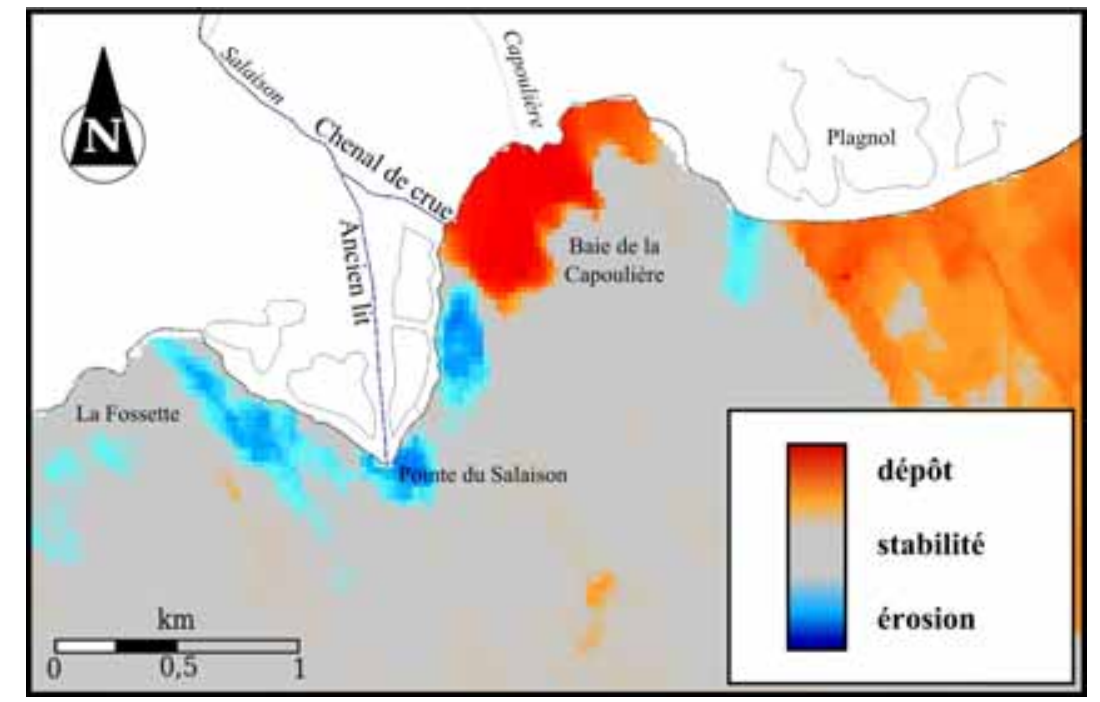

Figure 5. Evolution récente de la pointe du Salaison (étang de l'Or).

\section{4 $\underline{\text { Aspects quantitatifs }}$}

Bien que les incertitudes sur les bathymétries anciennes soient importantes, l'examen quantitatif des variations bathymétriques permet d'apprécier l’ordre de grandeur des vitesses d'évolution du système lagunaire. Les taux moyens d'évolution bathymétrique ainsi que les volumes moyens annuels accumulés, calculés à l'exclusion des zones comblées artificiellement, ont été estimés. Les résultats pour chacun des étangs sont présentés dans le tableau 3.

Les étangs d'Ingril, Pierre-Blanche, Arnel, Méjean, et Or présentent un comblement lent. Les étangs de Vic et Prévost sont globalement stables sur la période étudiée qui reste courte au regard des processus en jeu. L'étang du Grec quant à lui présente une tendance à l'érosion. Il s'agit également de la lagune la plus impactée par les comblements artificiels (figures 2 et 3), il est probable que des prélèvements de matériaux aient été effectués pour constituer les remblaiements ce qui expliquerait la tendance érosive observée. 
Lorsque des données intermédiaires sont disponibles, une bonne concordance est observée dans les taux d'évolution excepté pour l'étang de l'Or. Une sédimentation beaucoup plus forte y est observée dans la période ancienne. Vu l'écart important avec les autres lagunes, il est probable qu'une erreur dans l'acquisition des données sur cet étang soit à l'origine du phénomène. Pour l'étang de Pierre-Blanche, les résultats sont en accord avec les données géochronologiques disponibles, soit 2,7 $\pm 0,2 \mathrm{~mm} / \mathrm{an}$ (SABATIER et al., 2010). Il n'existe pas de données pour les autres lagunes du système. La tendance moyenne sur l'ensemble du complexe donne une vitesse de sédimentation globale de 1,3 $\pm 4 \mathrm{~mm} / \mathrm{an}$, soit une accumulation nette d'environ $93000 \mathrm{~m}^{3} / \mathrm{an}$. En se basant sur la profondeur moyenne du système lagunaire, et en supposant une stabilité des forçages actuels, cela entraînerait un comblement total des étangs dans 500 ans.

Tableau 3. Evolution sédimentaire des étangs Palavasiens (érosion négative).

\begin{tabular}{lllll}
\hline Lagune & $\mathbf{1 9 6 5 - 1 9 8 5}(\mathbf{m m} / \mathbf{a n})$ & $\mathbf{1 9 8 5 - 2 0 0 5}(\mathbf{m m} / \mathbf{a n})$ & $\mathbf{1 9 6 5 - 2 0 0 5}(\mathbf{m m} / \mathbf{a n})$ & volume $\left(\mathbf{m}^{3} / \mathbf{a n}\right)$ \\
\hline Ingril & - & - & $1,9 \pm 5$ & 9587 \\
Vic & - & - & $0,2 \pm 5$ & 11912 \\
Pierre-Bl. & $3,3 \pm 6$ & $2,8 \pm 2$ & $2,8 \pm 5$ & 7760 \\
Arnel & - & - & $2,0 \pm 5$ & 7065 \\
Prévost & $0,6 \pm 6$ & $-0,5 \pm 2$ & $0,0 \pm 5$ & 365 \\
Méjean & - & - & $1,1 \pm 5$ & 3289 \\
Grec & $0,0 \pm 6$ & $-2,5 \pm 2$ & $-1 \pm 5$ & -913 \\
Or & $10 \pm 6$ & $1,8 \pm 2$ & $7,2 \pm 5$ & 54255 \\
\hline
\end{tabular}

\section{Conclusions}

Cette analyse inédite de données topographiques et bathymétriques a permis de dresser un bilan de l'évolution récente du système lagunaire des étangs Palavasiens. Une tendance générale à la réduction de l'espace lagunaire est observée. L'évolution bathymétrique globale est assez lente, mais regroupe toutefois des fonctionnements distincts selon les étangs.

L'action anthropique apparaît comme un facteur important de cette évolution, elle est responsable de plus des $2 / 3$ des zones entièrement comblées au cours des 40 dernières années. Cette action peut être directe par la mise en œuvre de remblaiements. Elle peut également être indirecte par l'impact d'aménagements modifiant la dynamique du système, aménagements comme la canalisation de cours d'eau ou la protection des plages. La répartition des zones de dépôts suggère une contribution importante des apports du bassin versant dans la dynamique de comblement. Une dynamique marine active participe également au comblement de la lagune de Pierre-Blanche, secteur où le littoral n'est pas artificialisé contrairement au reste du site. 
Evolution morphologique récente d'un complexe lagunaire méditerranéen : le système des étangs Palavasiens (France) : 7.11

La forte incertitude sur les données bathymétriques anciennes et celle concernant l'élévation du niveau marin empêche d'estimer de façon fiable l'évolution future du système à partir de ces résultats. Un suivi plus précis de la dynamique lagunaire et des apports extérieurs (bassin versant et mer) s’avère nécessaire à l'avenir.

\section{Références bibliographiques}

ALLARD J., CHAUMILLON E., FENIES H. (2009). A synthesis of morphological evolutions and Holocene stratigraphy of a wave-dominated estuary: The Arcachon lagoon, SW France. Continental Shelf Research, $\mathrm{n}^{\circ}$ 29, pp 957-969. doi:10.1016/j.csr.2008.11.017

ANDREOSSY F. (1669). Carte du canal Royal de communication des mers en Languedoc.

BOURRIN F., DURRIEU DE MADRON X., LUDWIG W. (2006). Contribution to the study of coastal rivers and associated prodeltas to sediment supply in Gulf of Lions (NW Mediterranean Sea). Vie et Milieu, $\mathrm{n}^{\circ}$ 56(4), pp 307-314.

BARUSSEAU J.P., GIRESSE P., PLANCHAIS N., RADAKOVITCH O. (1992). La sédimentation lagunaire des derniers siècles en Languedoc-Roussillon : données sédimentologiques, isotopiques et palynologiques. Vie et Milieu, $\mathrm{n}^{\circ}$ 42(3-4), pp 307-320.

BIRD E.C.F. (1994). Physical settings and geomporphology of coastal lagoons. In Coastal Lagoon Processes. B. Kjerfve, pp 9-39. doi:10.1016/S0422-9894(08)70007-2

CABANES C., CAZENAVE A., LE PROVOST C. (2001). Sea Level Rise During Past 40 Years Determined from Satellite and in Situ Observations. Science, $\mathrm{n}^{\circ} 26$, pp 840-842. doi:10.1126/science.1063556

CALAFAT F., GOMIS D. (2009). Reconstruction of Mediterranean sea level fields for the period 1945-2000. Global and Planetary Change, $\mathrm{n}^{\circ}$ 66, pp 225-234. doi:10.1016/j.gloplacha.2008.12.015

CASSINI C. (1778). Carte du Royaume de France. Feuillets nº 57 \& 92.

CASTAINGS J. (2008). Etat de l'art des connaissances du phénomène de comblement des milieux lagunaires. Rapport Master 2 GLM, Université de Montpellier 2, Cepralmar. $100 \mathrm{p}$.

CATALIOTTI-VALDINA D. (1978). Modalités et bilan de la sédimentation pélitique dans un milieu margino-littoral: le complexe lagunaire de Bages-Sigean. Thèse, Université de Toulouse 3, 203 p.

CAZENAVE A., BONNEFOND P., MERCIER F., DOMINH K., TOUMAZOU V. (2002). Sea level variations in the Mediterranean Sea and Black Sea from satellite altimetry and tide gauges. Global and Planetary Change, $\mathrm{n}^{\circ} 34$, pp 59-86. doi:10.1016/S0921-8181(02)00106-6 
DEZILEAU L., SABATIER P., BLANCHEMANCHE P., JOLY B., SWINGEDOUW D., CASSOU C., CASTAINGS J., MARTINEZ P., VAN GRAFENSTEIN U. (2010). Intense storm activity during the Little Ice Age on the French Mediterranean Coast. Paleogeography, Paleoclimatology, Paleoecology, n² 299, pp 289-297.

DUBOUL-RAVAZET C., MARTIN R. (1981). La sédimentation Holocène dans trois étangs du littoral du Languedoc-Roussillon. Société Languedocienne de Géographie, $n^{\circ}$ 15(1-2), pp 69-86.

EHESS/CNRS \& BNF (2004). Des villages de Cassini aux communes d'aujourd'hui. [URL http://cassini.ehess.fr/cassini/fr/html/5_donnees.htm ]

GIEC (2007). Bilan 2007 des changements climatiques. Rapport d'évaluation du Groupe d'experts intergouvernemental sur l'évolution du climat (IV). 103 p.

MARTIN R. (1978). Evolution Holocène et actuelle des conditions de sédimentation dans le milieu lagunaire de Salses-Leucate. Thèse, Université de Toulouse 3, 210 p.

MOLINAROLI E., GUERZONI S., SARRETTA A., MASIOL M., PISTOLATO M. (2009). Thirty-year changes (1970-2000) in bathymetry and sediment texture recorded in the Lagoon of Venice sub-basins, Italy. Marine Geology, $\mathrm{n}^{\circ} 258$, pp 115-125. doi:10.1016/j.margeo.2008.12.001

MORTON R.A., WARD G. H., WHITE W. A. (2000). Rates of sediment supply and sea-level rise in a large coastal lagoon. Marine Geology, $\mathrm{n}^{\circ} 167$, pp 261-284. doi:10.1016/S0025-3227(00)00030-X

NICHOLS M.M. (1989). Sediment accumulation rates and relative sea-level rise in lagoons. Marine Geology, ${ }^{\circ}$ 88, pp 201-219. doi:10.1016/0025-3227(89)90098-4

PHLEGER F.B. (1969). Some general features of coastal lagoons. Coastal lagoons symposium, Novembre 1967, Mexico, pp 5-26.

RAYNAL O., BOUCHETTE F., CERTAIN R., SABATIER P., LOFI J., SERANNE M., DEZILEAU L., BRIQUEU L., FERER P., COURP T. (2010). Holocene evolution of a Languedocian lagoonal environnement controlled by inherited coastal morphology. Bulletin de la Société Géologique de France, n 181(2), pp 211-224.

SABATIER P. (2009). Reconstitution des évènements climatiques extrêmes (crues et tempêtes) au cours de l'Holocène dans le Golfe d'Aigues-Mortes (Sud de la France). Thèse, Université de Montpellier 2, 202 p.

SABATIER P., DEZILEAU L., BLANCHEMANCHE P., SIANI G., CONDOMINES M., BENTALEB I., PIQUES G. (2010). Holocene variations of radiocarbon reservoirs ages in a Mediterranean lagoonal system. Radiocarbon, $\mathrm{n}^{\circ}$ 52(1), pp 91-102.

SMNLR (2006). Bathymétrie des étangs Palavasiens et de l'étang de l'Or: méthodologie et moyens mis en ouvre. Rapport du Service Maritime et de Navigation du Languedoc-Roussillon. 24 p.

VAN DER WAL D., PYE K. (2003). The use of historical bathymetric charts in a GIS to assess morphological change in estuaries. The Geographical Journal, $\mathrm{n}^{\circ} 169(1)$, pp 21-31. 